\title{
О РАЗРЕШИМОСТИ НЕЛОКАЛЬНОЙ ЗАДАЧИ С ИНТЕГРАЛЬНЫМИ УСЛОВИЯМИ ВТОРОГО РОДА ДЛЯ ГИПЕРБОЛИЧЕСКОГО УРАВНЕНИЯ
}

\author{
В. А. Киричек \\ (Статья представлена членом редакционной коллегии А. П. Солдатовым) \\ Самарский национальный исследовательский университет имени академика С. П. Королева, \\ Самара, 443086, Россия \\ E-mail: Vitalya29@gmail.com
}

\begin{abstract}
Аннотация. В статье рассмотрена задача для гиперболического уравнения с интегральными условиями второго рода, содержащими в качестве внеинтегральных членов значения искомого решения на боковой границе. Нелокальные условия такого вида порождают значительные трудности при исследовании разрешимости задачи. Основным инструментом для доказательства этого утверждения являются априорные оценки в пространствах Соболева, получение которых стало возможным в результате применения метода, разработанного для случая одномерного гиперболического уравнения.
\end{abstract}

Ключевые слова: гиперболическое уравнение, нелокальная задача, интегральные условия, обобщенное решение, пространства Соболева.

Для цитирования: Киричек В. А. 2021. О разрешимости нелокальной задачи с интегральными условиями второго рода для гиперболического уравнения. Прикладная математика \& Физика. 53(4): 293-300. DOI 10.52575/2687-09592021-53-4-293-300.

\section{ON THE SOLVABILITY OF A NONLOCAL PROBLEM WITH INTEGRAL CONDITIONS OF THE SECOND KIND FOR HYPERBOLIC EQUATION}

\section{Vitaliya Kirichek}

(Article submitted by a member of the editorial board A. P. Soldatov)

Samara National Research University named after academician S. P. Korolev, Samara, 443086, Russia

E-mail: Vitalya29@gmail.com

Received December, 1, 2021

\begin{abstract}
In this paper we consider a problem for hyperbolic equation with integral conditions of the second kind containing as non-integral terms values of required solution on lateral boundary. Nonlocal conditions of this kind generate significant difficulties in the study of the solvability of the problem. The main tool for proving this statement is a priori estimates in Sobolev spaces, the obtaining of which became possible as a result of applying the method developed for the case of a one-dimensional hyperbolic equation.
\end{abstract}

Key words: hyperbolic equation, nonlocal problem, integral conditions, weak solution, Sobolev spaces.

For citation: Kirichek V.A. 2021. Solvability of a nonlocal problem with integral conditions of the second kind for hyperbolic equation. Applied Mathematics \& Physics. 54(3): 293-300. (in Russian) DOI 10.52575/2687-0959-2021-53-4-293-300.

1. Введение. В статье рассматривается задача отыскания решения в ограниченной области $Q_{T}=$ $(0, l) \times(0, T)$ гиперболического уравнения

$$
u_{t t}-\left(a(x, t) u_{x}\right)_{x}+c(x, t) u=f(x, t),
$$

удовлетворяющего начальным данным

$$
u(x, 0)=0, \quad u_{t}(x, 0)=0
$$

и нелокальным условиям

$$
\begin{aligned}
& u(0, t)+\int_{0}^{l} K_{1}(x) u(x, t) d x=0 \\
& u(l, t)+\int_{0}^{l} K_{2}(x) u(x, t) d x=0 .
\end{aligned}
$$


Задачи с нелокальными условиями для уравнений с частными производными уже несколько десятилетий являются объектом пристального внимания математиков. Большой интерес вызывают задачи с нелокальными интегральными условиями различных видов. Это связано с их разнообразными приложениями [11]. Многие процессы, изучаемые современным естествознанием, приводят при математическом моделировании к нелокальным задачам, которые часто оказываются более точными отражениями исследуемых процессов [16]. Отметим также тесную связь нелокальных и обратных задач $[3,14,20]$, в которых условие переопределения задается в интегральном виде. Нелокальные задачи для гиперболического уравнения, в том числе с интегральными условиями, изучались в работах $[1,2,5,6,8,9,10,13,15,17,18,19,21]$.

Исследования разрешимости нелокальных задач сталкивается с дополнительными трудностями, не свойственными самосопряженным краевым задачам ([2], [12]). Хорошо известно, что классические методы, применяемые для исследования разрешимости начально-краевых задач, неприменимы в случае нелокальных. К настоящему времени разработаны некоторые методы, позволяющие преодолеть трудности, возникающие из-за нелокальных условий. Эти методы различны, и выбор конкретного метода зависит от вида нелокальных условий. В этой статье мы рассматриваем пространственно нелокальные условия второго рода, содержащие след искомого решения на боковой границе.

Для обоснования разрешимости задачи с такими нелокальными условиями разработан метод [5], суть которого заключается в том, что вводится новая неизвестная функция специальным образом, в результате чего нелокальная задача сводится к обычной начально-краевой задаче для нагруженного уравнения относительно новой неизвестной функции, доказательство разрешимости которой получено методом продолжения по параметру.

Реализуя идею этого метода в одномерном случае, введем новую неизвестную функцию следующим образом:

$$
v(x, t)=u(x, t)+\int_{0}^{l} H(x, \xi) u(\xi, t) d \xi
$$

где $H(x, \xi)=\frac{1}{l}\left((l-x) K_{1}(\xi)+x K_{2}(\xi)\right)$.

В [4] доказана разрешимость поставленной задачи, однако только при выполнении весьма обременительных условий на функции $K_{i}(x)$. А именно, требуется, чтобы $K_{i}(0)=K_{i}(l)=0, \quad i=1,2$. Эти условия удалось ослабить, модифицировав метод сведения нелокальной задачи к краевой. Демонстрация предложенного метода и доказательство разрешимости нелокальной задачи и представляют основное содержание статьи.

Для обоснования разрешимости задачи с такими нелокальными условиями разработан метод [5], суть которого заключается в том, что вводится новая неизвестная функция специальным образом, в результате чего нелокальная задача сводится к обычной начально-краевой задаче для нагруженного уравнения относительно новой неизвестной функции, доказательство разрешимости которой получено методом продолжения по параметру.

Другой метод исследования разрешимости нелокальной задачи для уравнения (1) предложен в [10]. В этой статье показано, что условия (3) можно свести к динамическим нелокальным условиям, содержащим производные по пространственной переменной, что позволяет применить методы работы [9]. Однако в этом случае неизбежно возникают дополнительные требования на поводение решения на границе.

Ставя своей целью доказательство разрешимости задачи в пространстве $W_{2}^{1}\left(Q_{T}\right)$, дальнейшие исследования мы проводим с помощью техники, отличной от примененной как в [5], так и в [10]. Предложенная нами техника позволяет не только доказать разрешимость задачи, но и может оказаться полезной для нахождения приближенных решений.

В [4] доказана разрешимость поставленной задачи, однако только при выполнении весьма обременительных условий на функции $K_{i}(x)$. А именно, требуется, чтобы $K_{i}(0)=K_{i}(l)=0, i=1,2$. Эти условия удалось ослабить, модифицировав метод сведения нелокальной задачи к краевой.

Демонстрация предложенного модифицированного метода доказательства разрешимости нелокальной задачи и представляют основное содержание статьи.

2. Разрешимость задачи в $W_{2}^{1}\left(Q_{T}\right)$.

Определение 1.1. Функцию $u \in W_{2}^{1}\left(Q_{T}\right)$ будем называть обобщенным решением задачи (1)-(3), если для всех $w \in W_{2}^{1}\left(Q_{T}\right), w(x, T)=0, w(0, t)=w(l, t)=0$ выполняется тождество

$$
\int_{0}^{T} \int_{0}^{l}\left(-u_{t} w_{t}+a u_{x} w_{x}+c u w\right) d x d t=\int_{0}^{T} \int_{0}^{l} f(x, t) w(x, t) d x d t
$$

$u(x, 0)=0$ и (3)выполняются в смысле равенств в $L_{2}(0, T)$. 
Теорема 1.1. Пусть выполняются следующие условия:

$$
\begin{gathered}
a, a_{t}, a_{x}, c, \in C\left(\bar{Q}_{T}\right), \\
K_{i} \in C^{2}\left(\bar{Q}_{T}\right), K_{2}(0, t)=K_{1}(l, t)=0,2 l \int_{0}^{l}\left(K_{1}^{2}+K_{2}^{2}\right) d x<1 \forall t \in[0, T], \\
f, f_{t} \in L_{2}\left(Q_{T}\right) .
\end{gathered}
$$

Тогда существует единственное обобщенное решение задачи (1)-(3).

Доказательство. Доказательство состоит из нескольких этапов:

1. Сведение к краевой задаче для нагруженного уравнения.

2. Доказательство разрешимости краевой задачи для нагруженного уравнения.

3. Доказательство разрешимости задачи (1)-(3).

Приступим к доказательству теоремы.

1. Пусть $u(x, t)$ - решение задачи (1)-(3). Введем новую неизвестную функцию, положив

$$
v(x, t)=u(x, t)+\int_{0}^{x} K_{2}(\xi) u(\xi, t) d \xi+\int_{x}^{l} K_{1}(\xi) u(\xi, t) d \xi
$$

Тогда $v(x, t)$ удовлетворяет уравнению

$$
\begin{gathered}
v_{t t}-\left(a v_{x}\right)_{x}+c v-\int_{0}^{x} H_{1}(x, \xi) u(\xi, t) d \xi-\int_{x}^{l} H_{2}(x, \xi) u(\xi, t) d \xi \\
+b(x) u(x, t)-\alpha u(0, t)+\beta u(l, t)=F(x, t),
\end{gathered}
$$

где обозначено

$$
\begin{gathered}
H_{1}(x, \xi)=\left(K_{2 \xi} a\right)_{\xi}-K_{2}(\xi)[c(\xi)-c(x)] ; H_{2}(x, \xi)=\left(K_{1 \xi} a\right)_{\xi}-K_{1}(\xi)[c(\xi)-c(x)], \\
b(x)=\left(K_{2} a\right)_{x}-\left(K_{1} a\right)_{x}+a\left[K_{2 x}-K_{1 x}\right], \\
F(x, t)=f(x, t)-\int_{0}^{x} K_{2}(\xi) f(\xi, t) d \xi-\int_{x}^{l} K_{1}(\xi) f(\xi, t) d \xi, \\
\alpha=K_{2}^{\prime}(0) a(0), \quad \beta=K_{1}^{\prime}(l) a(l) .
\end{gathered}
$$

Заметим, что

$$
v(x, 0)=v_{t}(x, 0)=0, \quad v(0, t)=0, \quad v(l, t)=0 .
$$

Таким образом, мы приходим к краевой задаче:

Найти в $Q_{T}$ решение уравнения (6), удовлетворяющее условиям (7).

Заметим, что мы получили в результате введения новой неизвестной функции нагруженное уравнение. Вместо того чтобы выразить функцию $u(x, t)$ через $v(x, t)$ из (5), будем искать пару функций $(u, v)$.

Обозначим

$$
\begin{gathered}
W_{2,0}^{1}\left(Q_{T}\right)=\left\{v(x, t): v \in W_{2}^{1}\left(Q_{T}\right), v(0, t)=v(l, t)=0\right\}, \\
\hat{W}_{2,0}^{1}\left(Q_{T}\right)=\left\{\eta(x, t): \eta \in W_{2,0}^{1}\left(Q_{T}\right), \eta(x, T)=0\right\} .
\end{gathered}
$$

Определение 2. Пару функиий $(u, v)$ будем называть обобщенным решением задачи (6)-(7), если и $\in$ $W_{2}^{1}\left(Q_{T}\right), v \in W_{2,0}^{1}\left(Q_{T}\right), v(x, 0)=0$, для любой $\eta \in \hat{W}_{2,0}^{1}\left(Q_{T}\right)$ выполняется тождество

$$
\begin{gathered}
\int_{0}^{T} \int_{0}^{l}\left(-v_{t} \eta_{t}+a v_{x} \eta_{x}+c v \eta\right) d x d t=\int_{0}^{T} \int_{0}^{l} \eta(x, t) \int_{0}^{x} H_{1}(x, \xi) u(\xi, t) d \xi d x d t \\
+\int_{0}^{T} \int_{x}^{l} \eta(x, t) \int_{x}^{l} H_{2}(x, \xi) u(\xi, t) d \xi d x d t \\
+\int_{0}^{T} \int_{0}^{l} \eta(x, t)[b(x) u(x, t)-\alpha u(0, t)+\beta u(l, t)] d x d t \\
+\int_{0}^{T} \int_{0}^{l} F(x, t) \eta(x, t) d x d t
\end{gathered}
$$


а функиии и, г связань соотношением

$$
v(x, t)=u(x, t)+\int_{0}^{x} K_{2}(\xi) u(\xi, t) d \xi+\int_{x}^{l} K_{1}(\xi) u(\xi, t) d \xi
$$

2. Покажем, что при выполнении условий теоремы существует единственное обобщенное решение задачи (6)-(7).

Будем искать приближенное решение задачи (6)-(7). Положим $u^{0}=0$ и определим $\left(u^{n}, v^{n}\right)$ следующим образом:

$$
\begin{gathered}
\int_{0}^{T} \int_{0}^{l}\left(-v_{t}^{n} \eta_{t}+a v_{x}^{n} \eta_{x}+c v^{n} \eta\right) d x d t-\int_{0}^{T} \int_{0}^{l} \eta(x, t) \int_{0}^{x} H_{1}(x, \xi) u^{n-1}(\xi, t) d \xi d x d t \\
-\int_{0}^{T} \int_{0}^{l} \eta(x, t) \int_{x}^{l} H_{2}(x, \xi) u^{n-1}(\xi, t) d \xi d x d t \\
+\int_{0}^{T} \int_{0}^{l} \eta(x, t)\left[b(x) u^{n-1}(x, t)-\alpha u^{n-1}(0, t)+\beta u^{n-1}(l, t)\right] d x d t \\
=\int_{0}^{T} \int_{0}^{l} F(x, t) \eta(x, t) d x d t \\
v^{n}(x, t)=u^{n}(x, t)+\int_{0}^{x} K_{2}(\xi) u^{n}(\xi, t) d \xi+\int_{x}^{l} K_{1}(\xi) u^{n}(\xi, t) d \xi .
\end{gathered}
$$

Так как $u_{0}=0$, то для $v^{1}$ имеем

$$
\int_{0}^{T} \int_{0}^{l}\left(-v_{t}^{1} \eta_{t}+a v_{x}^{1} \eta_{x}+c v^{1} \eta\right) d x d t=\int_{0}^{T} \int_{0}^{l} F \eta d x d t
$$

Это означает, что $v^{1}(x, t)$ представляет собой обобщенное решение первой начально-краевой задачи для уравнения

$$
v_{t t}-\left(a v_{x}\right)_{x}+c v=F(x, t) .
$$

Известно ([7], с. 213-215), что это решение единственно и справедливо неравенство $\left\|v^{1}\right\|_{W_{2,0}^{1}}\left(Q_{T}\right) \leq$ $C\|F\|_{L_{2}\left(Q_{T}\right)}$. Кроме того, если $F_{t} \in L_{2}\left(Q_{T}\right)$, то $v^{1} \in W_{2}^{2}\left(Q_{T}\right)$ ([7], с. 216-219).

Теперь мы можем найти $u^{1}(x, t)$ из (11), так как при выполнении условий теоремы 1 (11) имеет единственное решение. Это решение принадлежит тому же пространству, что и $v^{1}(x, t)$, значит, $u^{1} \in$ $W_{2}^{1}\left(Q_{T}\right)$.

Затем находим $v^{2}(x, t)$ из (11) как решение первой начально-краевой задачи для уравнения (12) с правой частью

$$
F_{1}(x, t)=F(x, t)+\int_{0}^{x} H_{1} u^{1} d \xi+\int_{x}^{l} H_{2} u^{1} d \xi-b(x) u^{1}(x, t)+\alpha u^{1}(0, t)-\beta u^{1}(l, t) .
$$

Продолжив этот процесс, мы найдем $u^{n}(x, t)$ и $v^{n}(x, t)$. Условия теоремы гарантируют, что для каждого $n F_{n}, F_{n t} \in L_{2}\left(Q_{T}\right)$.

Таким образом, последовательность $\left(u^{n}, v^{n}\right)$ построена.

Покажем, что эта последовательность сходится при $n \rightarrow \infty$ в $W_{2,0}^{1}$ и ее предел $(u, v)$ является искомым обобщенным решением задачи (6)-(7). Для этого получим априорные оценки.

Обозначим $z^{n}=v^{n+1}-v^{n}, r^{n}=u^{n+1}-u^{n}$. Из (10) и (11) имеем

$$
\begin{gathered}
\int_{0}^{T} \int_{0}^{l}\left(-z_{t}^{n} \eta_{t}+a z_{x}^{n} \eta_{x}+c z^{n} \eta\right) d x d t=\int_{0}^{T} \int_{0}^{l} \eta(x, t) \int_{0}^{x} H_{1}(x, \xi) r^{n-1}(\xi, t) d \xi d x d t \\
\quad+\int_{0}^{T} \int_{0}^{l} \eta(x, t) \int_{x}^{l} H_{2}(x, \xi) r^{n-1}(\xi, t) d \xi d x d t \\
-\int_{0}^{T} \int_{0}^{l} \eta(x, t)\left[b(x) r^{n-1}(x, t)-\alpha r^{n-1}(0, t)+\beta r^{n-1}(l, t)\right] d x d t \\
z^{n}(x, t)=r^{n}(x, t)+\int_{0}^{x} K_{2}(\xi) r^{n}(\xi, t) d \xi+\int_{x}^{l} K_{1}(\xi) r^{n}(\xi, t) d \xi .
\end{gathered}
$$


Начнем с (13). В силу условий теоремы существуют положительные числа $\sigma_{1}, \sigma_{2}$ такие, что $\max _{[0, l]}\left|K_{i}(x)\right| \leq$ $\sigma_{i}$. Пусть $\sigma=\max \left\{\sigma_{1}, \sigma_{2}\right\}$ и $1-2 l \sigma>0$. Из (13) легко следует

$$
\left\|r^{n}\right\|_{L_{2}\left(Q_{T}\right)} \leq\left\|z^{n}\right\|_{L_{2}\left(Q_{T}\right)}+\left\|\int_{0}^{x} K_{2} r^{n} d \xi\right\|_{L_{2}\left(Q_{T}\right)}+\left\|\int_{x}^{l} K_{1} r^{n} d \xi\right\|_{L_{2}\left(Q_{T}\right)} .
$$

Тогда

$$
\left\|r^{n}\right\|_{L_{2}\left(Q_{T}\right)} \leq \frac{1}{1-2 \sigma l}\left\|z^{n}\right\|_{L_{2}\left(Q_{T}\right)}
$$

Из равенств $z_{t}^{n}=r_{t}^{n}+\int_{0}^{x} K_{2} r_{t}^{n} d \xi+\int_{x}^{l} K_{1} r_{t}^{n} d \xi$ и $z_{x}^{n}=r_{x}^{n}+\left(K_{2}-K_{1}\right) r^{n}$ получаем

$$
\begin{gathered}
\left\|r_{t}^{n}\right\|_{L_{2}\left(Q_{T}\right)} \leq \frac{1}{1-2 \sigma l}\left\|z_{t}^{n}\right\|_{L_{2}\left(Q_{T}\right)}, \\
\left\|r_{x}^{n}\right\|_{L_{2}\left(Q_{T}\right)} \leq\left\|z_{x}^{n}\right\|+\frac{2 \sigma}{1-2 \sigma l}\left\|z^{n}\right\|_{L_{2}\left(Q_{T}\right)} .
\end{gathered}
$$

Следовательно,

$$
\left\|r^{n}\right\|_{W_{2}^{1}\left(Q_{T}\right)}^{2} \leq A\left\|z^{n}\right\|_{W_{2}^{1}\left(Q_{T}\right)}^{2},
$$

где $A=\max \left\{\frac{1+4 \sigma^{2}}{(1-2 \sigma l)^{2}}, 2\right\}$.

Заметим, что мы ищем $z^{n}$ как решение уравнения

$$
z_{t t}^{n}-\left(a z_{x}^{n}\right)_{x}+c z^{n}=F^{n}(x, t)
$$

с правой частью

$$
\begin{gathered}
F^{n}(x, t)=\int_{0}^{x} H_{1}(x, \xi) r^{n-1}(\xi, t) d \xi+\int_{x}^{l} H_{2}(x, \xi) r^{n-1}(\xi, t) d \xi-b(x) r^{n-1}(x, t) \\
+\alpha r^{n-1}(0, t)-\beta r^{n-1}(l, t),
\end{gathered}
$$

удовлетворяющее условиям

$$
z^{n}(0, t)=z^{n}(l, t)=z^{n}(x, 0)=z_{t}^{n}(x, 0)=0 .
$$

Так как $F^{n}, F_{t}^{n} \in L_{2}\left(Q_{T}\right)$, то $z^{n} \in W_{2}^{2}\left(Q_{T}\right)$ и мы можем вывести следующее соотношение:

$$
\begin{gathered}
\int_{0}^{l}\left[\left(z_{t}^{n}(x, \tau)\right)^{2}+a(x)\left(z_{x}^{n}(x, \tau)\right)^{2}\right] d x=-2 \int_{0}^{\tau} \int_{0}^{l} c z^{n} z_{t}^{n} d x d t+2 \int_{0}^{\tau} \int_{0}^{l} F^{n} z_{t}^{n} d x d t . \\
\int_{0}^{l}\left[\left(z_{t}^{n}(x, \tau)\right)^{2}+a(x)\left(z_{x}^{n}(x, \tau)\right)^{2}\right] d x \leq A_{1} \int_{0}^{\tau} \int_{0}^{l}\left[\left(z^{n}\right)^{2}+\left(z_{t}^{n}\right)^{2}+\left(z_{x}^{n}\right)^{2}\right] d x d t \\
+c(\varepsilon) \int_{0}^{\tau} \int_{0}^{l}\left(z_{t}^{n}\right)^{2} d x d t+\varepsilon \int_{0}^{\tau} \int_{0}^{l}\left(F^{n}(x, t)\right)^{2} d x d t .
\end{gathered}
$$

Оценим последнее слагаемое с помощью неравенства Коши - Буняковского и неравенства, связывающего значения функции на границе и во внутренних точках области [9]

$$
\left(r^{n-1}\left(\xi_{i}, t\right)\right)^{2} \leq 2 l \int_{0}^{l}\left(r_{x}^{n-1}\right)^{2} d x+\frac{2}{l} \int_{0}^{l}\left(r^{n-1}\right)^{2} d x, \quad \xi_{1}=0, \xi_{2}=l .
$$

Тогда

$$
\begin{gathered}
\int_{0}^{\tau} \int_{0}^{l}\left(\int_{0}^{x} H_{1} r^{n-1} d \xi\right)^{2} d x d t+\int_{0}^{\tau} \int_{0}^{l}\left(\int_{x}^{l} H_{2} r^{n-1} d \xi\right)^{2} d x d t \\
\leq l^{2}\left(\sigma_{1}+\sigma_{2}\right) \int_{0}^{\tau} \int_{0}^{l}\left(r^{n-1}\right)^{2} d x d t \\
\int_{0}^{\tau} \int_{0}^{l}\left(b r^{n-1}\right)^{2} d x d t \leq b_{0}^{2} \int_{0}^{\tau} \int_{0}^{l}\left(r^{n-1}\right)^{2} d x d t ; \\
\alpha^{2} \int_{0}^{\tau} \int_{0}^{l}\left(r^{n-1}(0, t)\right)^{2} d x d t+\beta^{2} \int_{0}^{\tau} \int_{0}^{l}\left(r^{n-1}(l, t)\right)^{2} d x d t \leq
\end{gathered}
$$




$$
\leq 2\left(\alpha^{2}+\beta^{2}\right) \int_{0}^{\tau} \int_{0}^{l}\left[l^{2}\left(r_{x}^{n-1}\right)^{2}+\left(r^{n-1}\right)^{2} d x d t .\right.
$$

Прибавим к (15) неравенство

$$
\int_{0}^{l}\left(z^{n}(x, \tau)\right)^{2} d x \leq \tau \int_{0}^{\tau} \int_{0}^{l}\left(z_{t}^{n}(x, t)\right)^{2} d x d t .
$$

Для его вывода заметим, что имеет место представление $z^{n}(x, \tau)=\int_{0}^{\tau} z_{t}^{n}(x, t) d t+z^{n}(x, 0)$. Так как $z^{n}(x, 0)=0$, то $z^{n}(x, \tau)=\int_{0}^{\tau} z_{t}^{n}(x, t) d t$. Возведя обе части этого равенства в квадрат и применяя неравенство Коши - Буняковского в правой его части, получим

$$
\left(z^{n}(x, t)\right)^{2}=\left(\int_{0}^{\tau} z_{t}^{n}(x, t) d t\right)^{2} \leq\left(\int_{0}^{\tau} d t \int_{0}^{\tau}\left(z_{t}^{n}\right)^{2} d t\right)=\tau \int_{0}^{\tau}\left(z_{t}^{n}\right)^{2} d t,
$$

интегрируя которое по $x$ и убеждаемся в справедливости данного неравенства .

Тогда получим

$$
\begin{gathered}
\int_{0}^{l}\left[\left(z^{n}(x, \tau)\right)^{2}+\left(z_{t}^{n}(x, \tau)\right)^{2}+a(x, \tau)\left(z_{x}^{n}(x, \tau)\right)^{2}\right] d x \leq A_{2} \int_{0}^{\tau} \int_{0}^{l}\left[\left(z^{n}\right)^{2}+\left(z_{t}^{n}\right)^{2}+\left(z_{x}^{n}\right)^{2}\right] d x d t \\
+\varepsilon A_{3} \int_{0}^{\tau} \int_{0}^{l}\left[\left(r^{n-1}\right)^{2}+\left(r_{t}^{n-1}\right)^{2}+\left(r_{x}^{n-1}\right)^{2}\right] d x d t
\end{gathered}
$$

где $A_{1}, A_{2}, A_{3}$ зависят только от $a_{0}, a_{1}, c_{0}, b_{0}, \sigma_{1}, \sigma_{2}$ и не зависят от $n$.

Применив лемму Гронуолла и проинтегрировав результат по $(0, T)$, получим

$$
\left\|z^{n}\right\|_{W_{2}^{1}\left(Q_{T}\right)}^{2} \leq \varepsilon B\left\|r^{n-1}\right\|_{W_{2}^{1}\left(Q_{T}\right)}^{2},
$$

где $B=T A_{3} e^{A_{2} T}$. Таким образом, из (14) и (16)

$$
\left\|z^{n}\right\|_{w_{2}^{1}\left(Q_{T}\right)}^{2} \leq A B \varepsilon\left\|z^{n-1}\right\|_{w_{2}^{1}\left(Q_{T}\right)}^{2}, \quad\left\|r^{n}\right\|_{w_{2}^{1}\left(Q_{T}\right)}^{2} \leq A B \varepsilon\left\|r^{n-1}\right\|_{w_{2}^{1}\left(Q_{T}\right)}^{2} .
$$

Выберем $\varepsilon$ так, чтобы $0<\varepsilon A B<1$. Тогда последовательность $\left\{u^{n}, v^{n}\right\}$ сходится в $W_{2}^{1}\left(Q_{T}\right)$ к некоторой паре (причем единственной) $(u, v) \in W_{2}^{1}\left(Q_{T}\right)$.

Переходя при $n \rightarrow \infty$ в (10), (11), убеждаемся в том, что $(u, v)$ является искомым решением задачи (6) $-(7)$.

3. Разрешимость задачи (1)-(3). Если $(u, v)$ - решение задачи (6)-(7), то в соответствии с определением обобщенного решения (8) и (9) выполняются.

Подставив $v(x, t)$, представленную формулой (9) в (8), после несложных преобразований получим (3). Очевидно, (4) также выполнено.

Следовательно, $u(x, t)$ есть решение задачи (1)-(3).

Теорема доказана полностью.

\section{Список литературы}

1. Гордезиани Д. Г., Авалишвили Г. А. 2000. Решения нелокальных задач для одномерных колебаний среды. Математическое моделирование, 12(1): 94-103.

2. Ионкин Н. И. 1977. Решение одной краевой задачи теории теплопроводности с неклассическим краевым условием. Дифференциальные уравнения, 13(2): 294-301.

3. Камынин В. Л. 2013. Обратная задача определения младшего коэффициента в параболическом уравнении при условии интегрального наблюдения. Математические заметки, 94(2): 207-217.

4. Киричек В. А. 2020. О разрешимости одной задачи с нелокальными условиями для гиперболического уравнения. Вестник Самарского университета. Естественнонаучная серия, 26(4): 36-43.

5. Кожанов А. И., Пулькина Л. С. 2006. О разрешимости краевых задач с нелокальным граничным условием интегрального вида для многомерных гиперболических уравнений. Дифференциальные уравнения, 42(9): 1166-1179. 
6. Корзюк В. И., Козловская И. С., Наумовец С. Н. 2019. Классическое решение задачи для одномерного волнового уравнения с интегральными условиями второго рода. Дифференциальные уравнения, 55(3): 361-369.

7. Ладыженская О. А. 1973. Краевые задачи математической физики. М., Наука, 407.

8. Пулькина Л. С. 2008. Начально-краевая задача с нелокальным граничным условием для многомерного гиперболического уравнения. Дифференциальные уравнения, 44(8): 1084-1089.

9. Пулькина Л. С. 2012. Краевые задачи для гиперболического уравнения с нелокальными условиями 1 и 2-го рода. Известия вузов. Математика, 56(4): 74-83.

10. Пулькина Л. С., Савенкова А. Е. 2016. Нелокальная задача с интегральными условиями второго рода для гиперболического уравнения. Вестник Самарского университета. Естественнонаучная серия, 2016(1-2): 33-45.

11. Самарский А. А. 1980. О некоторых проблемах современной теории дифференциальных уравнений. Дифференциальные уравнения 16(11): 1221-1228.

12. Скубачевский А. Л., Стеблов Г. М. 1991. О спектре дифференциальных операторов с областью определения, не плотной в $L_{2}(0,1)$. Доклады Академии Наук СССР, 321(6): 1158-1163.

13. Ashyralyev A., Aggez N. 2012. On the Solution of Multipoint NBVP for Hyperbolic Equation with Integral Condition. Malaysian Journal of Mathematical Sciences, 6(S): 111-121.

14. Ashyralyev A., Sharifov Y. A. 2013. Optimal control problems for impulsive systems with integral boundary conditions. Electronic journal of differential equations, 2013(80): 1-11.

15. Avalishvili G., Avalishvili M., Gordeziani D. 2011. On Integral Nonlocal Boundary Value Problems for some Partial Differential Equations. Bulletin of the Georgian National Academy of Sciences, 5(1): 31-37.

16. Bažant Z. P., Jirásek M. 2002. Nonlocal Integral Formulation of Plasticity And Damage: Survey of Progress. American Society of Civil Engineers. Journal of Engineering Mechanics, 128(11): 1119-1149.

17. Beilin S. A. 2006. On a mixed nonlocal problem for a wave equation. Electronic journal of differential equations, 2006(103): 1-10.

18. Bouziani A. 2002. On the solvability of parabolic and hyperbolic problems with a boundary integral condition. IJMMS journal, 31(4): 201-213.

19. Cannon J. R. 1963. The solution of the heat equation subject to the specification of energy. Quarterly of Applied Mathematics, 21(2): 155-160.

20. Cannon J. R., Lin Y. 1990. An Inverse Problem of Finding a Parameter in a Semi-linear Heat Equation. Journal of Mathematical Analysis and Applications, 145: 470-484.

21. Pulkina L. S. 2020. Nonlocal Problems for Hyperbolic Equation from the viewpoint of strongly regular boundary conditions. Electronic journal of differential equations, 28.

\section{References}

1. Gordeziani D. G., Avalishvili G. A. 2000. Solutions of nonlocal problems for one-dimensional oscillations of the medium. Mathematical Modeling, 12(1): 94-103. (In Russian)

2. Ionkin N. I. 1977. A Solution of Certain Boundary-Value Problem of Heat Conduction with Nonclassical Boundary Condition. Differential Equations, 13(2): 294-301.(in Russian)

3. Kamynin V. L. 2013. The inverse problem of determining the lower-crder coefficient in parabolic equations with integral observation, Mathematical Notes, 94(2): 207-217. (In Russian)

4. Kirichek V. A. 2020 About solvability of one problem with nonlocal conditions for hyperboliic equation. Vestnik of Samara University. Natural Science Series, 26(4): 36-43. (in Russian)

5. Kozhanov A. I., Pulkina L. S. 2006. On the Solvability of Boundary Value Problems with a Nonlocal Boundary Condition of Integral Form for Multidimentional Hyperbolic Equations. Differential Equations, 42(9): 1233-1246. (in Russian) 
6. Korzyuk V. I., Kozlovskaya I. S., Naumavets S. N. 2019. Classical Solution of a Problem with Integral Congitions of the Second Kind for the One-Dimensional Wave Equation. Differential Equations, 55(3): 353-362. (in Russian)

7. Ladyzhenskaya O. A. 1973. Kraevye zadachi matematicheskoi fiziki Boundary-value problems of mathematical physics. M., Nauka, 407.

8. Pulkina L. S. 2008. Initial-boundary value problem with a nonlocal boundary condition for a multidimensional hyperbolic equation. Differential Equation, 44(8): 1119-1125. (In Russian)

9. Pulkina L. S. 2012. Boundary value problems for a hyperbolic equation with nonlocal conditions of the I and II kind. Russian Mathematics, 56(4): 62-69. (in Russian)

10. Pulkina L. S., Savenkova A. E. 2016. A problem with second kind integral conditions for hyperbolic equation. Vestnik of Samara University. Natural science series. 2016(1-2): 33-45.

11. Samarskii A. A. 1980. Some problems of the theory of differential equations. Differentsial'nye Uravneniya, 16(11): 1925-1935.

12. Skubachevskii A. L., Steblov G. M. 1991. On the spectrum of differential operators with a domain that is not dense in $L_{2}(0,1)$. Doklady Akademii Nauk SSSR, 321(6): 1158-1163.(In Russian)

13. Ashyralyev A., Aggez N. 2012. On the Solution of Multipoint NBVP for Hyperbolic Equation with Integral Condition. Malaysian Journal of Mathematical Sciences, 6(S): 111-121.

14. Ashyralyev A., Sharifov Y. A. 2013. Optimal control problems for impulsive systems with integral boundary conditions. Electronic journal of differential equations, 2013(80): 1-11.

15. Avalishvili G., Avalishvili M., Gordeziani D. 2011. On Integral Nonlocal Boundary Value Problems for some Partial Differential Equations. Bulletin of the Georgian National Academy of Sciences, 5(1): 31-37.

16. Bažant Z. P., Jirásek M. 2002. Nonlocal Integral Formulation of Plasticity And Damage: Survey of Progress. American Society of Civil Engineers. Journal of Engineering Mechanics, 128(11): 1119-1149.

17. Beilin S. A. 2006. On a mixed nonlocal problem for a wave equation. Electronic journal of differential equations, 2006(103): 1-10.

18. Bouziani A. 2002. On the solvability of parabolic and hyperbolic problems with a boundary integral condition. IJMMS journal, 31(4): 201-213.

19. Cannon J. R. 1963. The solution of the heat equation subject to the specification of energy. Quarterly of Applied Mathematics, 21(2): 155-160.

20. Cannon J. R., Lin Y. 1990. An Inverse Problem of Finding a Parameter in a Semi-linear Heat Equation. Journal of Mathematical Analysis and Applications, 145: 470-484.

21. Pulkina L. S. 2020. Nonlocal Problems for Hyperbolic Equation from the viewpoint of strongly regular boundary conditions. Electronic journal of differential equations, 28.

Конфликт интересов: о потенциальном конфликте интересов не сообщалось.

Conflict of interest: no potential conflict of interest related to this article was reported.

Получена 01.12.2021

\section{СВЕДЕНИЯ ОБ АВТОРЕ}

Киричек Виталия Александровна - ассистент кафедры дифференциальных уравнений и теории управления, Самарский национальный исследовательский университет имени академика С. П. Королева.

http://orcid.org/0000-0001-9817-863X

Московское шоссе, 34, 443086, Самара, Россия

E-mail: Vitalya29@gmail.com

\section{INFORMATION ABOUT THE AUTHOR}

Kirichek Vitaliya Alexandrovna - assistant of the Department of Differential Equations and Control Theory, Samara National Research University, 34, Moskovskoye shosse, 443086, Samara, Russian Federation. 